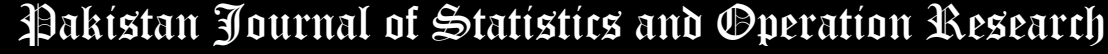

\section{Extended Reciprocal Rayleigh Distribution: Copula, Properties and Real Data Modeling}

\author{
Rezk H.
}

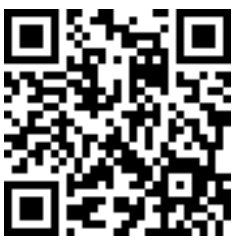

Mathematical Sciences Department, College of Science, Princess Nourah bint Abdulrahman University, Riyadh, Saudi Arabia. Department of Statistics, Al-Azhar University, Cairo, Egypt, hodaragab2009@yahoo.com

\begin{abstract}
A new extension of the reciprocal Rayleigh distribution is introduced. Simple type copula-based construction is presented for deriving and many bivariate and multivariate type distributions of the reciprocal Rayleigh model. The new reciprocal Rayleigh model generalizes another three reciprocal Rayleigh distributions. The performance of the estimation method is assessed using a graphical simulation study.
\end{abstract}

Key Words: Reciprocal Rayleigh Distribution; Clayton Copula; Morgenstern Family; Moments; Estimation; Odd Log-Logistic Family; Graphical Simulation.

Mathematical Subject Classification: 62N01; 62N02; 62E10.

\section{Introduction}

A random variable (RV) $Z$ is said to have the reciprocal Rayleigh (RR) distribution if its survival function (SF) is given by

$$
S_{\beta}(z)=1-G_{\beta}(z)=1-\left.\exp \left(-\beta^{2} z^{-2}\right)\right|_{z \geq 0},
$$

where $\beta>0$ and $G_{\beta}(z)$ refer to the cumulative function (CDF). The density function (PDF) corresponding (1) can be expressed as

$$
g_{\beta}(z)=\left.2 \beta^{2} z^{-3} \exp \left(-\beta^{2} z^{-2}\right)\right|_{z \geq 0}
$$

The RR model is a special case from the well-known Reciprocal Weibull (RW) model. Recently, Cordeiro et al. (2016) proposed and studied a new class called the generalized odd log-logistic-G (GOLL-G) with two extra shape parameters $a_{1}>0$ and $a_{2}>0$. For an arbitrary baseline CDF $G_{\underline{\Psi}}(z)$, the SF of the GOLL-G family is given by

$$
S_{a_{1}, a_{2}, \underline{\Psi}}(z)=1-F_{a_{1}, a_{2}, \underline{\Psi}}(z)=1-\frac{G_{\underline{\Psi}}(z)^{a_{1} a_{2}}}{G_{\underline{\Psi}}(z)^{a_{1} a_{2}}+\left[1-G_{\underline{\Psi}}(z)^{a_{2}}\right]^{a_{1}},}
$$

The PDF corresponding to (3) is given by

$$
f_{a_{1}, a_{2}, \underline{\underline{Y}}}(z)=\frac{a_{1} a_{2} g_{\underline{\Psi}}(z) G_{\underline{\Psi}}(z)^{a_{1} a_{2}-1}\left[1-G_{\underline{\Psi}}(z)^{a_{2}}\right]^{a_{1}-1}}{\left\{G_{\underline{\Psi}}(z)^{a_{1} a_{2}}+\left[1-G_{\underline{\Psi}}(z)^{a_{2}}\right]^{a_{1}}\right\}^{2}},
$$

where $g_{\underline{\underline{ }}}(z)=d G_{\underline{\Psi}}(z) / d x$. For $a_{2}=1$ we get the OLL-G family (Gleaton and Lynch (2006)). For $a_{1}=1$ we get the Proportional reversed hazard rate (ProRHR) family (Gupta and Gupta (2007)). Here, we define a new RW 
model based on Cordeiro et al. (2016) called GOLL-RR model and then provide some plots of its PDF and hazard rate function (HRF) $\left[h_{a_{1}, a_{2}, \beta}(z)\right]$. The SF of the GOLL-RR is given by

$$
S_{a_{1}, a_{2}, \beta}(z)=1-F_{a_{1}, a_{2}, \beta}(z)=1-\frac{\exp \left(-a_{1} a_{2} \beta^{2} z^{-2}\right)}{\exp \left(-a_{1} a_{2} \beta^{2} z^{-2}\right)+\left\{1-\exp \left(-a_{2} \beta^{2} z^{-2}\right)\right\}^{a_{1}}} .
$$

The PDF corresponding to (5) is given by

$$
f_{a_{1}, a_{2}, \beta}(z)=\frac{a_{1} a_{2} 2 \beta^{2} z^{-3} \exp \left(-a_{1} a_{2} \beta^{2} z^{-2}\right)\left\{1-\exp \left(-a_{2} \beta^{2} z^{-2}\right)\right\}^{a_{1}-1}}{\left(\exp \left(-a_{1} a_{2} \beta^{2} z^{-2}\right)+\left\{1-\exp \left(-a_{2} \beta^{2} z^{-2}\right)\right\}^{a_{1}}\right)^{2}} .
$$

The HRF for the new model can be get from $f_{a_{1}, a_{2}, \beta}(z) /\left[1-F_{a_{1}, a_{2}, \beta}(z)\right]$. Table 1 provides some sub-models of the GOLL-RR model.

Table 1: Sub-models of the GOLL-RR model

\begin{tabular}{cccccc}
\hline$a_{1}$ & $a_{2}$ & $\beta$ & Reduced model & Reduced CDF & Author \\
\hline 1 & & ProRH-RR & $\exp \left(-a_{2} \beta^{2} z^{-2}\right)$ & Gusmao et al. (2011) \\
& 1 & OLL-RR & $\frac{\exp \left(-a_{1} \beta^{2} z^{-2}\right)}{\left(\begin{array}{c}\exp \left(-a_{1} \beta^{2} z^{-2}\right) \\
+\left[1-\exp \left(-\beta^{2} z^{-2}\right)\right]^{a_{1}}\end{array}\right\}}$ & Yousof et al. (2018) \\
& & & $\exp \left(-\beta^{2} z^{-2}\right)$ & Trayer (1964) \\
\hline
\end{tabular}

Fig. 1 give some plots of the GOLL-RR PDF and HRF. From Fig. 1 (left panel) the new GOLL-RR PDF can be a unimodal-PDF with right skewed and symmetric shape. From Fig. 1 (left panel) we note that the new GOLL-RR HRF can be upside-down-HRF with different useful shapes.
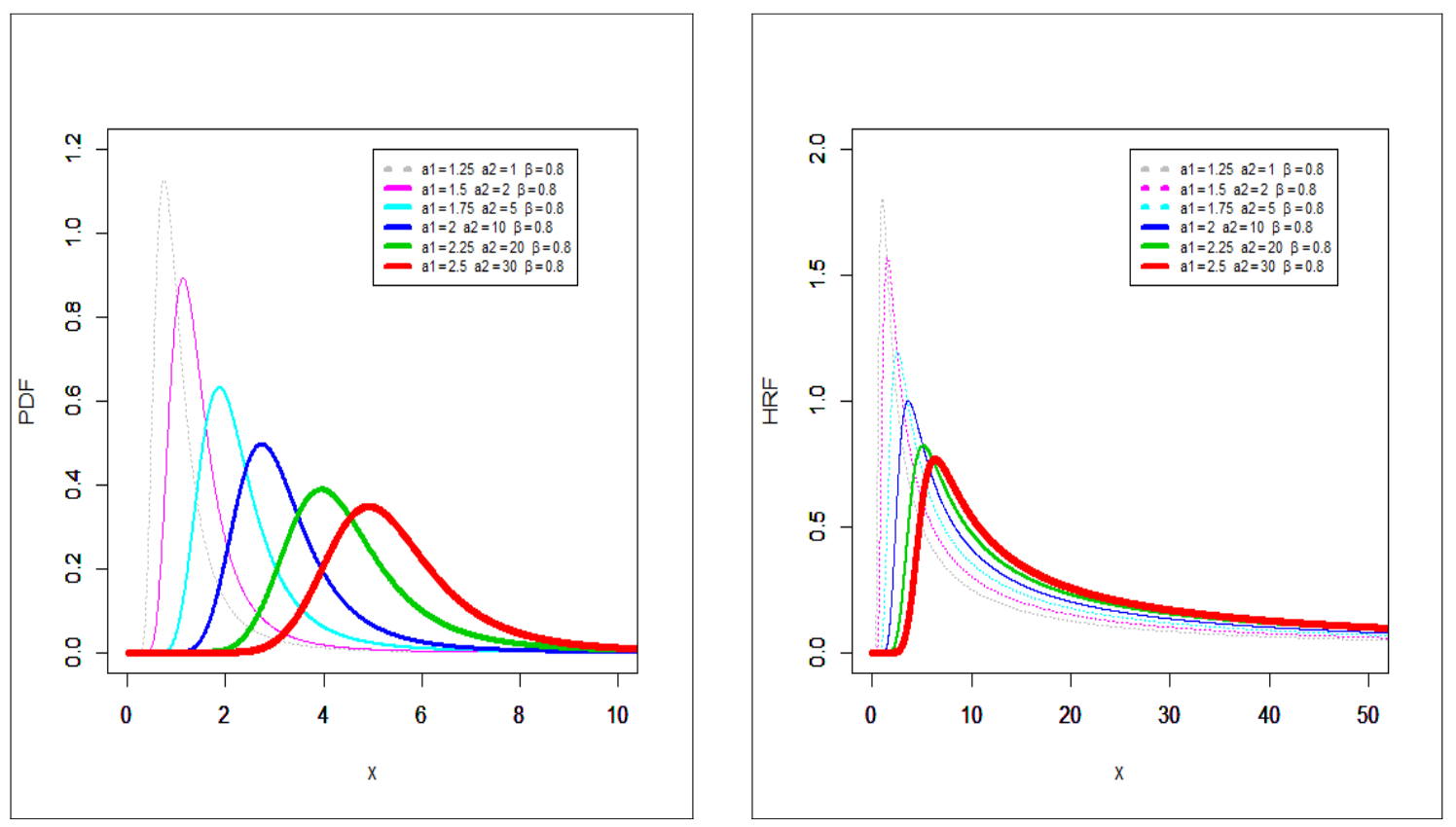

Figure 1: Plots of the GOLL-RR PDF and HRF. 
The quantile function $(\mathrm{QF})$ of $z$ (by inverting (5)), say $z_{\mathrm{Q}}=Q(\mathrm{Q})=F^{-1}(\mathrm{Q})$, as

$$
z_{Q}=\beta /\left(-\ln \left\{\left[\frac{\left(\frac{\mathrm{Q}}{1-\mathrm{Q}}\right)^{\frac{1}{a_{1}}}}{1+\left(\frac{\mathrm{Q}}{1-\mathrm{Q}}\right)^{\frac{1}{a_{1}}}}\right]^{\frac{1}{a_{2}}}\right\}\right)^{\frac{1}{2}}
$$

\section{Simple type copula}

\subsection{Via Morgenstern family}

The CDF of the Morgenstern family

where

$$
\begin{aligned}
& \left.F_{\lambda}\left(z_{1}, z_{2}\right)\right|_{(|\lambda| \leq 1)}=F_{a_{1}, a_{2}, \beta_{1}}\left(z_{1}\right) F_{b_{1}, b_{2}, \beta_{2}}\left(z_{2}\right) \\
& \times\left\{1+\lambda\left[1-F_{a_{1}, a_{2}, \beta_{1}}\left(z_{1}\right)\right]\left[1-F_{b_{1}, b_{2}, \beta_{2}}\left(z_{2}\right)\right]\right\},
\end{aligned}
$$

and

$$
1-F_{a_{1}, a_{2}, \beta_{1}}\left(z_{1}\right)=1-\frac{\exp \left(-a_{1} a_{2} \beta_{1}^{2} z_{1}^{-2}\right)}{\exp \left(-a_{1} a_{2} \beta_{1}^{2} z_{1}^{-2}\right)+\left\{1-\exp \left(-a_{2} \beta_{1}^{2} z_{1}^{-2}\right)\right\}^{a_{1}}}
$$

$$
1-F_{b_{1}, b_{2}, \beta_{2}}\left(z_{2}\right)=1-\frac{\exp \left(-b_{1} b_{2} \beta_{2}^{2} z_{1}^{-2}\right)}{\exp \left(-b_{1} b_{2} \beta_{1}^{2} z_{2}^{-2}\right)+\left\{1-\exp \left(-b_{2} \beta_{1}^{2} z_{2}^{-2}\right)\right\}^{b_{1}}}
$$

then we have a 7-dimension parameter model as

$$
\begin{aligned}
& \left.F_{\lambda}\left(z_{1}, z_{2}\right)\right|_{(|\lambda| \leq 1)}=\frac{\exp \left(-a_{1} a_{2} \beta_{1}^{2} z_{1}^{-2}\right)}{\exp \left(-a_{1} a_{2} \beta_{1}^{2} z_{1}^{-2}\right)+\left\{1-\exp \left(-a_{2} \beta_{1}^{2} z_{1}^{-2}\right)\right\}^{a_{1}}} \\
& \times \frac{\exp \left(-b_{1} b_{2} \beta_{2}^{2} z_{1}^{-2}\right)}{\exp \left(-b_{1} b_{2} \beta_{1}^{2} z_{2}^{-2}\right)+\left\{1-\exp \left(-b_{2} \beta_{1}^{2} z_{2}^{-2}\right)\right\}^{b_{1}}} \\
& \times\left\{\begin{array}{c}
1+\lambda\left[1-\frac{\exp \left(-a_{1} a_{2} \beta_{1}^{2} z_{1}^{-2}\right)}{\exp \left(-a_{1} a_{2} \beta_{1}^{2} z_{1}^{-2}\right)+\left\{1-\exp \left(-a_{2} \beta_{1}^{2} z_{1}^{-2}\right)\right\}^{a_{1}}}\right] \\
{\left[1-\frac{\exp \left(-b_{1} b_{2} \beta_{2}^{2} z_{1}^{-2}\right)}{\exp \left(-b_{1} b_{2} \beta_{1}^{2} z_{2}^{-2}\right)+\left\{1-\exp \left(-b_{2} \beta_{1}^{2} z_{2}^{-2}\right)\right\}^{b_{1}}}\right]}
\end{array}\right\} .
\end{aligned}
$$

\subsection{Via clayton copula} The bivariate extension

Let

$$
H\left(c_{1}, c_{2}\right) \mid\left(\lambda_{1}+\lambda_{2}\right) \geq 0=\left[c_{1}^{-\left(\lambda_{1}+\lambda_{2}\right)}+c_{2}^{-\left(\lambda_{1}+\lambda_{2}\right)}-1\right]^{-\frac{1}{\lambda_{1}+\lambda_{2}}} .
$$

Assume that $X \sim \operatorname{GOLL}-R R\left(a_{1}, a_{2}, \beta_{1}\right)$ and $Y \sim \operatorname{GOLL}-R R\left(b_{1}, b_{2}, \beta_{2}\right)$. Then, setting

and

$$
c_{1}=c_{1}\left(a_{1}, a_{2}, \beta_{1}\right)=\frac{\exp \left(-a_{1} a_{2} \beta_{1}^{2} x^{-2}\right)}{\exp \left(-a_{1} a_{2} \beta_{1}^{2} x^{-2}\right)+\left\{1-\exp \left(-a_{2} \beta_{1}^{2} x^{-2}\right)\right\}^{a_{1}}}
$$

$$
c_{2}=c_{2}\left(b_{1}, b_{2}, \beta_{2}\right)=\frac{\exp \left(-b_{1} b_{2} \beta_{2}^{2} y^{-2}\right)}{\exp \left(-b_{1} b_{2} \beta_{1}^{2} y^{-2}\right)+\left\{1-\exp \left(-b_{2} \beta_{1}^{2} y^{-2}\right)\right\}^{b_{1}}},
$$

the associated CDF bivariate GOLL-RR type distribution will be 


$$
H(x, y)=\left(\begin{array}{c}
{\left[\frac{\exp \left(-a_{1} a_{2} \beta_{1}^{2} x^{-2}\right)}{\exp \left(-a_{1} a_{2} \beta_{1}^{2} x^{-2}\right)+\left\{1-\exp \left(-a_{2} \beta_{1}^{2} x^{-2}\right)\right\}^{a_{1}}}\right]^{-\left(\lambda_{1}+\lambda_{2}\right)}} \\
+\left[\frac{\exp \left(-b_{1} b_{2} \beta_{2}^{2} y^{-2}\right)}{\exp \left(-b_{1} b_{2} \beta_{1}^{2} y^{-2}\right)+\left\{1-\exp \left(-b_{2} \beta_{1}^{2} y^{-2}\right)\right\}^{b_{1}}}\right]^{-\left(\lambda_{1}+\lambda_{2}\right)} \\
-1
\end{array}\right)^{-\frac{1}{\lambda_{1}+\lambda_{2}}}
$$

The Multivariate extension

The $m$-dimensional type extension can be derived as

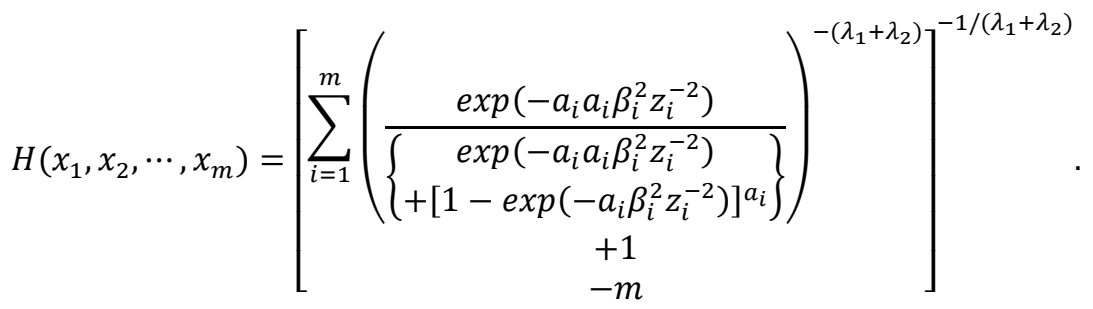

\section{Properties}

\subsection{Asymptotics}

Let

$$
\tau=\inf \left\{\left.z\right|_{G(z ; \underline{\Psi})>0}\right\}
$$

then

$$
\begin{gathered}
\left.F_{a_{1}, a_{2}, \beta}(z) \sim \exp \left(-a_{1} a_{2} \beta^{2} z^{-2}\right)\right|_{z \rightarrow \tau} \\
\left.f_{a_{1}, a_{2}, \beta}(z) \sim 2 a_{1} a_{2} \beta^{2} z^{-3} \exp \left(-a_{1} a_{2} \beta^{2} z^{-2}\right)\right|_{z \rightarrow \tau} \\
\left.h_{a_{1}, a_{2}, \beta}(z) \sim 2 a_{1} a_{2} \beta^{2} z^{-3} \exp \left(-a_{1} a_{2} \beta^{2} z^{-2}\right)\right|_{z \rightarrow \tau} . \\
1-\left.F_{a_{1}, a_{2}, \beta}(z) \sim\left(a_{2}\left\{1-\exp \left(-\beta^{2} z^{-2}\right)\right\}\right)^{a_{1}}\right|_{z \rightarrow \infty}, \\
\left.f_{a_{1}, a_{2}, \beta}(z) \sim 2 a_{1} a_{2}^{a_{1}} \beta^{2} z^{-3} \exp \left(-\beta^{2} z^{-2}\right)\left\{1-\exp \left(-\beta^{2} z^{-2}\right)\right\}^{a_{1}-1}\right|_{z \rightarrow \infty}
\end{gathered}
$$

and

$$
\left.h_{a_{1}, a_{2}, \beta}(z) \sim \frac{2 a_{1} \beta^{2} z^{-3} \exp \left(-\beta^{2} z^{-2}\right)}{1-\exp \left(-\beta^{2} z^{-2}\right)}\right|_{z \rightarrow \infty}
$$

\subsection{Representations}

Based on Cordeiro et al. (2016), the PDF in (6) can be expressed as

$$
f(z)=\sum_{\kappa=0}^{\infty} \Upsilon_{\kappa} \pi_{(1+\kappa)}(z ; \beta),
$$

where

$$
\begin{aligned}
& \Upsilon_{\kappa}=\frac{a_{1} a_{2}}{1+\kappa} \sum_{\zeta_{1}, \zeta_{2}=0}^{\infty} \sum_{\kappa=0}^{\zeta_{3}}(-1)^{\zeta_{2}+\kappa+\zeta_{3}}\left(\begin{array}{c}
-2 \\
\zeta_{1}
\end{array}\right) \\
& \times\left(\begin{array}{c}
-a_{1}\left(\zeta_{1}+1\right) \\
\zeta_{2}
\end{array}\right)\left(\begin{array}{c}
a_{1} a_{2}\left(\zeta_{1}+1\right)+a_{2} \zeta_{2}-1 \\
\zeta_{3}
\end{array}\right)\left(\begin{array}{c}
\zeta_{3} \\
\kappa
\end{array}\right),
\end{aligned}
$$

and $\pi_{(1+\kappa)}(z ; \beta)$ is the PDF of the RR model with scale parameter $\beta \sqrt{(1+\kappa)}$ also the CDF of $Z$ becomes

$$
F(z)=\sum_{\kappa=0}^{\infty} \Upsilon_{\kappa} \Pi_{(1+\kappa)}(z ; \beta),
$$


where $\Pi_{(1+\kappa)}(z ; \beta)$ is the $\mathrm{CDF}$ of the RR distribution.

\subsection{Moments}

The $r^{t h}$ ordinary moment of $Z$ is given by

then we obtain

$$
\mu_{r}^{\prime}=E\left(z^{r}\right)=\int_{-\infty}^{\infty} z^{r} f(z) d z
$$

$$
\mu_{r}^{\prime}=\sum_{\kappa=0}^{\infty} \Upsilon_{\kappa} \beta^{r}(1+\kappa)^{\frac{r}{2}} \Gamma\left(1-\frac{r}{2}\right), \forall 2>r
$$

where

$$
\left.\Gamma\left(1+\omega_{1}\right)\right|_{\left(\omega_{1} \in R^{+}\right)}=\omega_{1} !=\prod_{h=0}^{\omega_{1}-1}\left(\omega_{1}-h\right) .
$$

The $r^{\text {th }}$ incomplete moment (IM) can be obtained as

$$
\vartheta_{r}(t)=\int_{-\infty}^{t} \quad z^{r} f(z) d z=\sum_{\kappa=0}^{\infty} \quad Y_{\kappa} \int_{-\infty}^{t} \quad z^{r} \pi_{(1+\kappa)}(z ; \beta) d z,
$$

then

$$
\vartheta_{r}(t)=\sum_{\kappa=0}^{\infty} \gamma_{\kappa} \beta^{r}[(1+\kappa)]^{\frac{r}{2}} \gamma\left(1-\frac{r}{2},[(1+\kappa)]\left(\frac{\beta}{t}\right)^{2}\right), \forall 2>r
$$

where $\gamma\left(\omega_{1}, \omega_{2}\right)$ is the incomplete gamma function

$$
\begin{aligned}
& \left.\gamma\left(\omega_{1}, \omega_{2}\right)\right|_{\left(\omega_{1} \neq 0,-1,-2, \ldots\right)}=\int_{0}^{\omega_{2}} t^{\omega_{1}-1} \exp (-t) d t \\
& =\frac{\omega_{2}^{\omega_{1}}}{\omega_{1}}\left\{1 F_{1}\left[\omega_{1} ; \omega_{1}+1 ;-\omega_{2}\right]\right\} \\
& =\sum_{\kappa=0}^{\infty} \frac{(-1)^{\kappa}}{\kappa !\left(\omega_{1}+\kappa\right)} \omega_{2}^{\omega_{1}+\kappa},
\end{aligned}
$$

and $1 F_{1}[\because,, \cdot]$ is a confluent hypergeometric function (CHF). The first IM given by (11) with $r=1$ as

$$
\vartheta_{1}(t)=\sum_{\kappa=0}^{\infty} \Upsilon_{\kappa} \beta \sqrt{(1+\kappa)} \Gamma\left(1-\frac{1}{2},(1+\kappa)\left(\frac{\beta}{t}\right)^{2}\right) .
$$

The MGF $M_{z}(t)=E\left(e^{t z}\right)$ of $z$ can be derived from equation (8) as

$$
M_{z}(t)=\sum_{\kappa=0}^{\infty} \sum_{r=0}^{\infty}\left(t^{r} Y_{\kappa} / r !\right) \beta^{r}[(1+\kappa)]^{\frac{r}{2}} \Gamma\left(1-\frac{r}{2}\right), \forall 2>r
$$

The Lorenz (L) and Bonferroni (B) curves are defined by

and

$$
\mathrm{L}=\frac{\vartheta_{1}(t)}{\mu_{1}^{\prime}}
$$

respectively. Then, for the new $R R$ we have

$$
\mathrm{B}=\frac{\vartheta_{1}(t)}{\mu_{1}^{\prime} F(z)}=\frac{\mathrm{L}}{F(z)}
$$

$$
\mathrm{L}=\frac{\sum_{\kappa=0}^{\infty} \Upsilon_{\kappa} \beta \sqrt{(1+\kappa)} \Gamma\left(1-\frac{1}{2},(1+\kappa)\left(\frac{\beta}{t}\right)^{2}\right)}{\sum_{\kappa=0}^{\infty} \Upsilon_{\kappa} \beta \sqrt{(1+\kappa)} \Gamma\left(1-\frac{1}{2}\right)},
$$

and 


$$
\mathrm{B}=\frac{\sum_{\kappa=0}^{\infty} \Upsilon_{\kappa} \beta \sqrt{(1+\kappa)} \Gamma\left(1-\frac{1}{2},(1+\kappa)\left(\frac{\beta}{t}\right)^{2}\right)}{\sum_{\kappa=0}^{\infty} \Upsilon_{\kappa} \beta \sqrt{(1+\kappa)} \Gamma\left(1-\frac{1}{2}\right)}
$$

\subsection{Residual life and reversed residuals and their moments}

The $n^{\text {th }}$ moment of the residuals

Then, the $n^{\text {th }}$ moment is given by

$$
A_{n, z}(t)=E\left[\left.(z-t)^{n}\right|_{(z>t, n=1,2, \ldots)}\right] .
$$

$$
A_{n, z}(t)=\frac{\int_{t}^{\infty}(z-t)^{n} d F(z)}{1-F(t)}
$$

Therefore,

where

$$
A_{n, z}(t)=\frac{\beta^{n}}{1-F(t)} \sum_{\kappa=0}^{\infty} \zeta_{\kappa}[(1+\kappa)]^{\frac{n}{2}} \Gamma\left(1-\frac{n}{2},[(1+\kappa)]\left(\frac{\beta}{t}\right)^{2}\right), \forall 2>n,
$$

and

$$
\begin{gathered}
\zeta_{\kappa}=Y_{\kappa} \sum_{r=0}^{n}(-t)^{r}\left(\begin{array}{l}
n \\
r
\end{array}\right), \\
\left.\Gamma\left(\omega_{1}, \omega_{2}\right)\right|_{z>0}=\int_{\omega_{2}}^{\infty} t^{\omega_{1}-1} \exp (-t) d t
\end{gathered}
$$

The $n^{\text {th }}$ moment of the reversed residuals

$$
\Gamma\left(\omega_{1}, \omega_{2}\right)+\gamma\left(\omega_{1}, \omega_{2}\right)=\Gamma\left(\omega_{1}\right) .
$$

Then

$$
B_{n, z}(t)=E\left[\left.(t-z)^{n}\right|_{(z \leq t, t>0 \text { and } n=1,2, \ldots)}\right] .
$$

Then, the $n^{\text {th }}$ moment becomes

$$
B_{n, z}(t)=\frac{\int_{0}^{t}(t-z)^{n} d F(z)}{F(t)}
$$

where

$$
B_{n, z}(t)=\frac{\beta^{n}}{F(t)} \sum_{\kappa=0}^{\infty} \eta_{\kappa}[(1+\kappa)]^{\frac{n}{2}} \gamma\left(1-\frac{n}{2},(1+\kappa)\left(\frac{\beta}{t}\right)^{2}\right), \forall 2>n,
$$

\section{Maximum likelihood estimation (MLE)}

$$
\eta_{\kappa}=Y_{\kappa} \sum_{r=0}^{n}(-1)^{r}\left(\begin{array}{l}
n \\
r
\end{array}\right) t^{n-r}
$$

The log-likelihood function

$$
\begin{aligned}
& \ell=\ell(\Theta)=n \log \left(2 a_{1} a_{2} \beta^{2}\right)-(2+1) \sum_{i=1}^{n} \log \left(z_{i}\right)-a_{1} a_{2} \beta^{2} \sum_{i=1}^{n} z_{i}^{-2} \\
& +2 \sum_{i=1}^{n} \log \left\{\exp \left(-a_{1} a_{2} \beta^{2} z_{i}^{-2}\right)+\left[1-\exp \left(-a_{2} \beta^{2} z_{i}^{-2}\right)\right]^{a_{1}}\right\} \\
& +\left(a_{1}-1\right) \sum_{i=1}^{n} \log \left[1-\exp \left(-a_{2} \beta^{2} z_{i}^{-2}\right)\right] .
\end{aligned}
$$

The components of the "score vector" is available if needed. 


\section{Simulation studies}

We can perform the simulation experiments to assess of the finite sample behavior of the MLEs based on the following algorithm:

1.Use (7) to generate 1000 samples of size $n$ from the GOLL-RR model

2.Compute the MLEs for the 1000 samples.

3.Compute the SEs of the MLEs for the 1000 samples.

4.The standard errors (SEs) were computed by inverting the observed information matrix.

5.Compute the biases and MSEs for $h=a_{1}, a_{2}, \beta$.

We repeated these steps for $n=50,100, \ldots, 200$ with $a_{1}=1, a_{2}=1, \beta=1$, so computing biases $\left(\operatorname{Bias}_{h}(n)\right)$, mean squared errors $\left(M S E_{h}(n)\right)$ for $a_{1}, a_{2}, \beta$ and $n=50,100, \ldots, 200$. Fig.s 2, 3 and 4 give the biases and MSEs for $a_{1}, a_{2}$ and $\beta \forall n=50,100, \ldots, 200$ for the GOLL-RR model. Fig.s 2, 3 and 4 shows how the biases and MSEs vary with respect to $n$. The broken line in Fig. 4 corresponds to the biases being 0 . From Fig. 2, 3 and 4, the biases decrease to zero as $n \rightarrow \infty$, the MSEs for each parameter decrease to zero as $n \rightarrow \infty$.

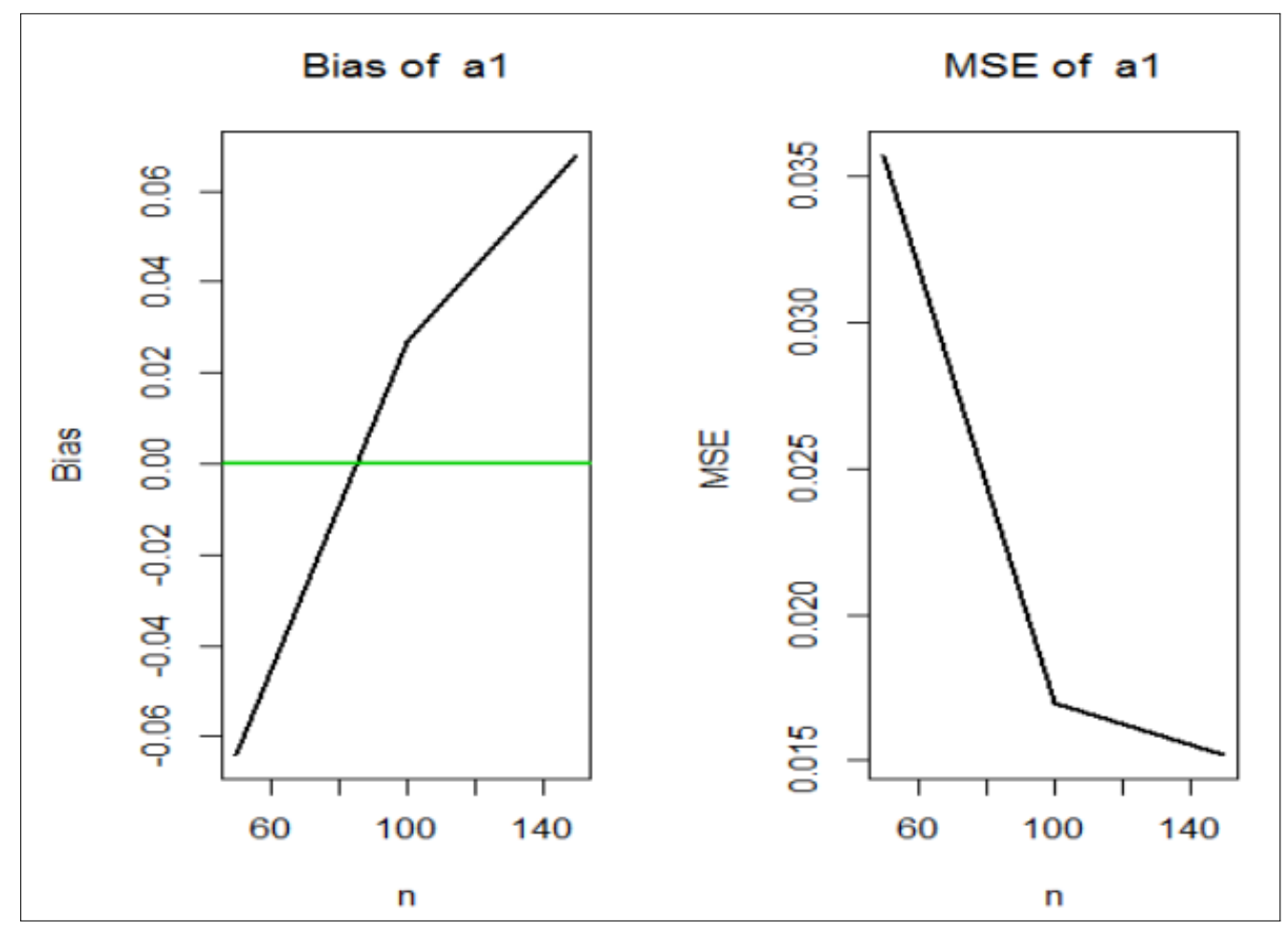

Fig. 2: Biases and MSEs for $a_{1}$ for the GOLL-RR model. 


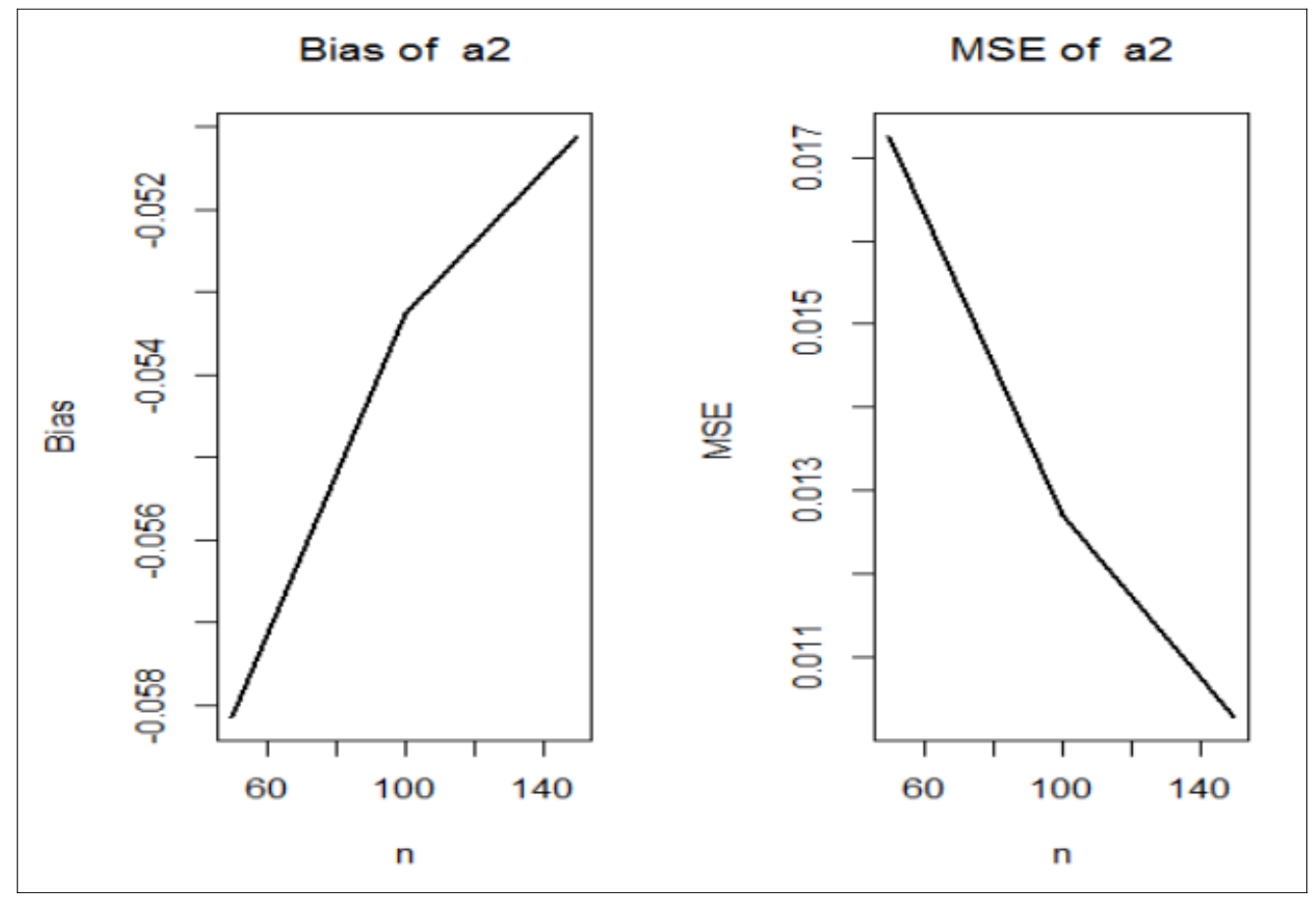

Fig. 3: Biases and MSEs for $a_{2}$ for the GOLL-RR model.

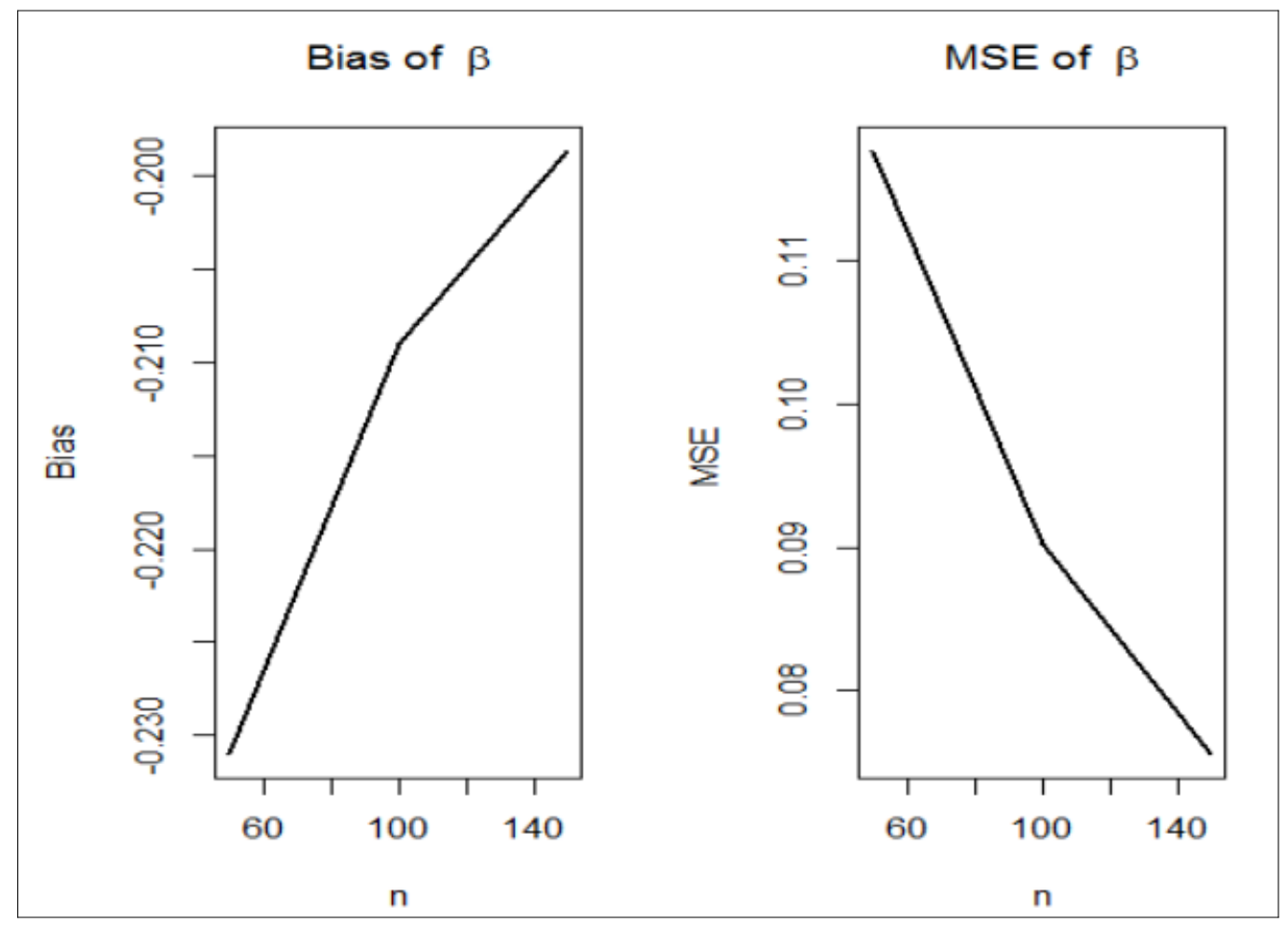

Fig. 4: Biases and MSEs for $\beta$ for the GOLL-RR model. 


\section{Real data modeling}

We consider the Cramér-Von Mises (C-V-M) and the Anderson-Darling (A-D) and the Kolmogorov-Smirnov (KS) statistic.

We compare the fits of the GOLL-RR distribution with other models such as RW, Kumaraswamy-RW (KFr), exponentiated RW (E-RW), beta-RW (B-RW), transmuted-RW (T-RW), Marshal-Olkin-RW (MO-RW) and McDonald-RW (Mc-RW) distributions given by:

E-RW:

B-RW:

$$
f(z)=a_{1} b \beta^{b} z^{-(b+1)} \exp \left[-(\beta / z)^{b}\right]\left\{1-\exp \left[-(\beta / z)^{b}\right]\right\}^{a_{1}-1} ;
$$

K-RW:

$$
f(z)=b \beta^{b} B^{-1}\left(a_{1}, c\right) z^{-(b+1)} \exp \left[-a_{1}(\beta / z)^{b}\right]\left\{1-\exp \left[-(\beta / z)^{b}\right]\right\}^{c-1} ;
$$

T-RW:

$$
f(z)=a_{1} c b \beta^{b} z^{-(b+1)} \exp \left[-a_{1}(\beta / z)^{b}\right]\left\{1-\exp \left[-a_{1}(\beta / z)^{b}\right]\right\}^{c-1} ;
$$

MO-RW:

$$
f(z)=b \beta^{b} z^{-(b+1)} \exp \left[-(\beta / z)^{b}\right]\left\{1+a_{1}-2 a_{1} \exp \left[-(\beta / z)^{b}\right]\right\} ;
$$

Mc-RW:

$$
f(z)=a_{1} b \beta^{b} z^{-(b+1)} \exp \left[-(\beta / z)^{b}\right]\left\{a_{1}+\left(1-a_{1}\right) \exp \left[-(\beta / z)^{b}\right]\right\}^{-2} ;
$$

OLLE-RW:

$$
\begin{gathered}
f(z)=a_{2} b \beta^{b} z^{-(b+1)} B^{-1}\left(a_{1}, c\right) \exp \left[-(\beta / z)^{b}\right] \\
\times\left\{\exp \left[-(\beta / z)^{b}\right]\right\}^{a_{1} a_{2}-1}\left\{1-\exp \left[-a_{2}(\beta / z)^{b}\right]\right\}^{c-1}
\end{gathered}
$$

$$
\begin{aligned}
& f(z)=a_{2} \beta b a^{b} z^{-(b+1)} \exp \left[-\beta(a / z)^{b}\right] \\
& \times\left(\exp \left[-\beta(a / z)^{b}\right]\left\{1-\exp \left[-\beta(a / z)^{b}\right]\right\}\right)^{-1+a_{2}} \\
& \times\left(\exp \left[-\beta a_{2}(a / z)^{b}\right]+\left\{1-\exp \left[-\beta(a / z)^{b}\right]\right\}^{a_{2}}\right)^{-2}
\end{aligned}
$$

The parameters of the above densities are all positive real numbers except for the T-RW distribution for which $\left|a_{1}\right| \leq 1$.

The $1^{\text {st }}$ data (see Nichols and Padgett (2006)). Fig. 6 gives the total time test (TTT) plot (see Aarset (1987)) for data set I. It indicates that the empirical HRFs of data sets I is increasing HRF (IHRF). The $2^{\text {nd }}$ data (see Smith and Naylor (1987)). Fig. 6 gives the TTT plot for data set II. It indicates that the empirical HRFs of data sets II is IHRF. The 3rd data set (wingo data). Fig. 7 gives the TTT plot for data set III. It indicates that the empirical HRFs of data sets III is increasing.

Many other useful real data sets can be found in Aryal and Yousof (2017), Merovci et al. (2017), Korkmaz et al. (2017), Hamedani et al. (2017), Alizadeh et al. (2017), Brito et al. (2017), Alizadeh et al. (2018), Korkmaz et al. (2018), Yousof et al. (2018a-d), Hamedani et al. (2018), Cordeiro et al. (2018), Hamedani et al. (2019), Ibrahim (2019), Nascimento et al. (2019), Ibrahim et al. (2019), Goual and Yousof (2019), Korkmaz et al. (2019), Alizadeh et al. (2019), Yousof et al. (2019) and Goual et al. (2020), Ibrahim (2020a,b), Yadav et al. (2020) and Ibrahim et al. (2020). 


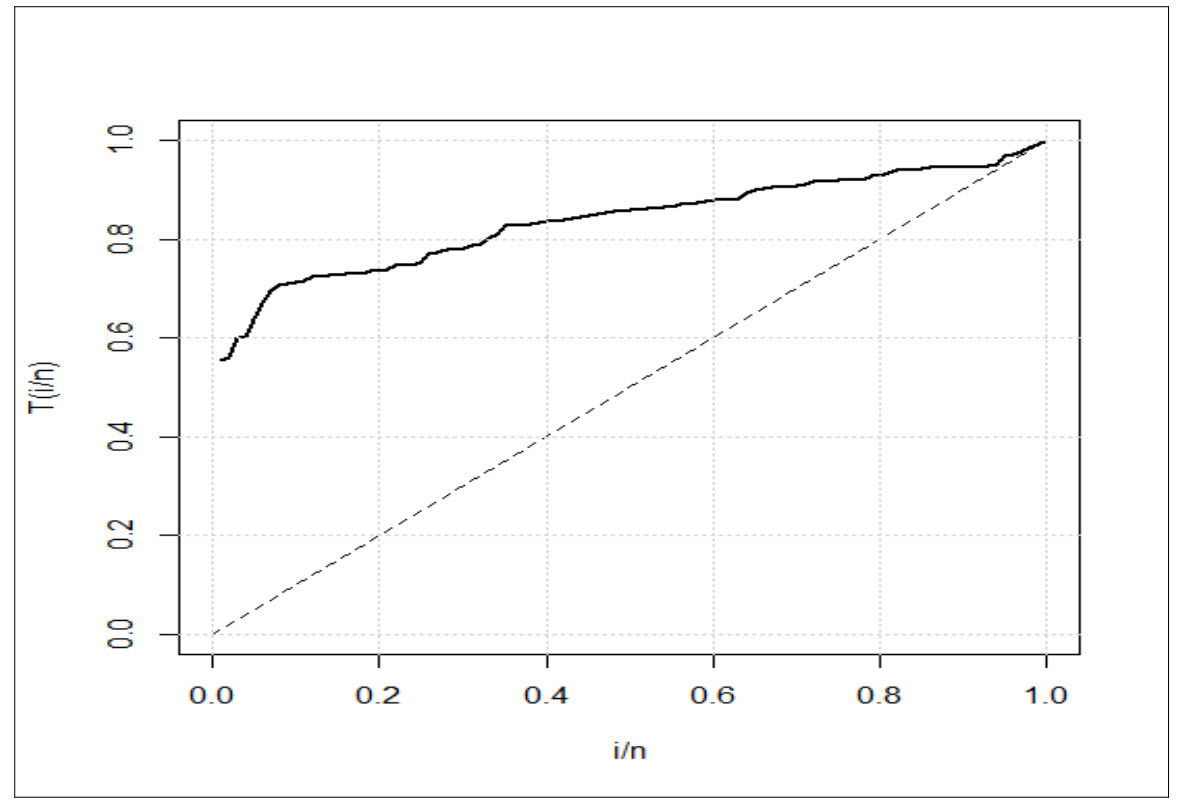

Fig. 5: TTT plot for data set I.

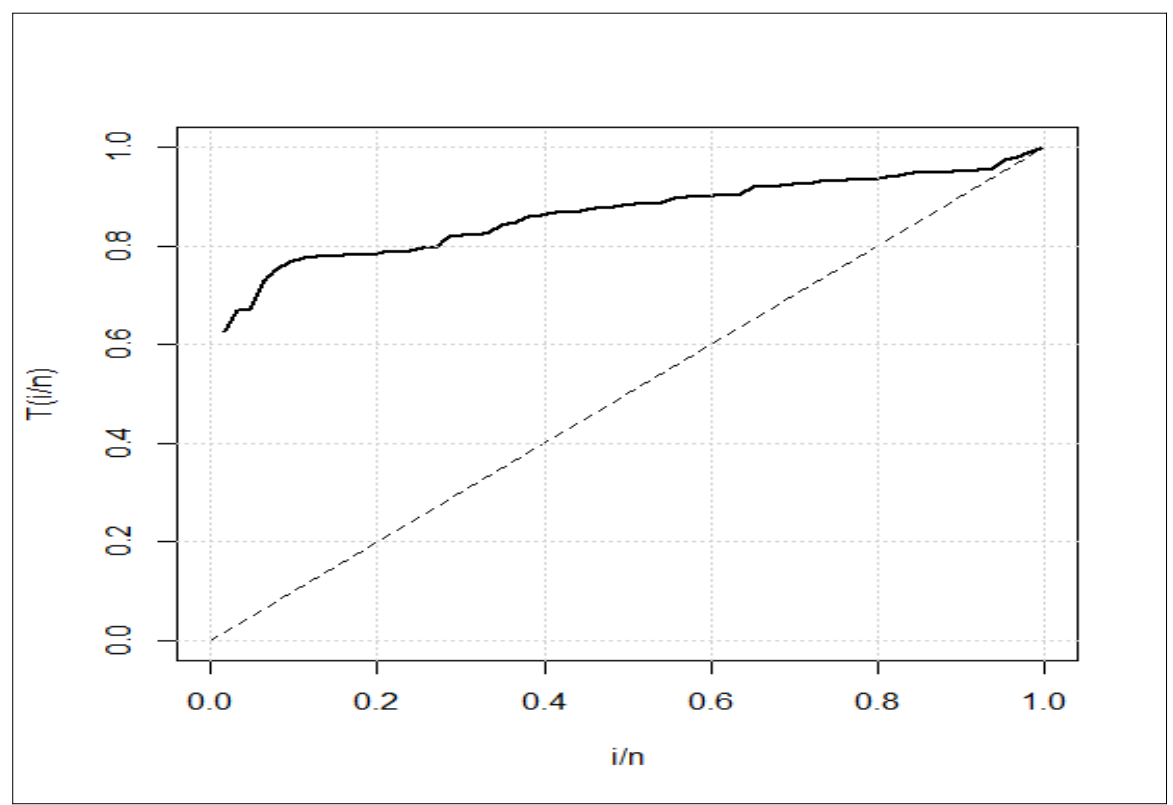

Fig. 6: TTT plot for data set II. 


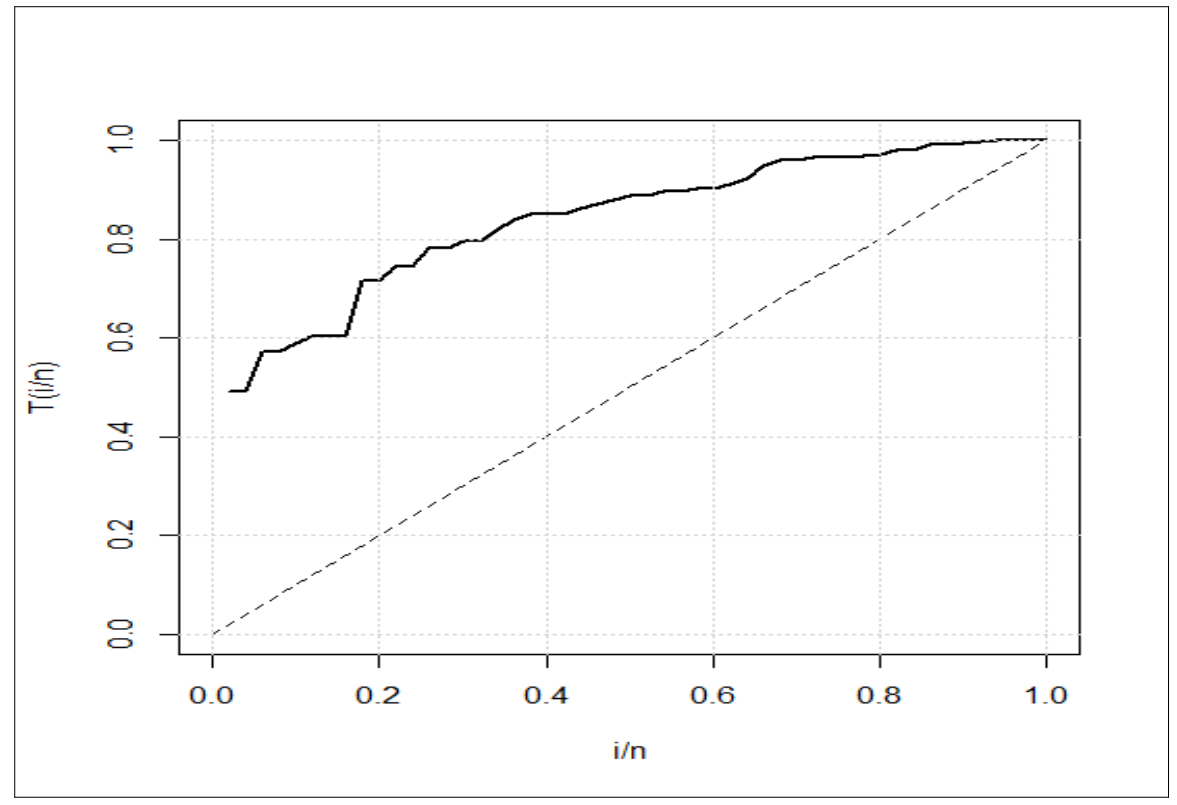

Fig. 7: TTT plot for data set III.

The statistics are presented in Tables 2, 4 and 6 for data sets I-III respectively. The MLEs and corresponding standard errors (SEs) are given in Tables 3, 5 and 7 for data sets I-III respectively. Fig. 5, 6 and 7 gives the estimated density (E-PDF), estimated CDF (E-CDF), P-P plot (P-P) and estimated HRF (E-HRF) for data set I-III respectively. The GOLL-RR distribution in Tables 2, 4 and 6 give the best results.

Table 2

\begin{tabular}{lllll}
\hline Model & \multicolumn{2}{l}{ Goodness of fit criteria } & K-S & P-value \\
\hline GOLL-RR & C-V-M & A-D & 0.05775 & 0.8926 \\
OLLE-RW & 0.0592 & 0.4845 & 0.5561 & $2.2 \times \mathrm{e}^{-16}$ \\
OLLE-RR & 0.1203 & 0.9639 & 0.6550 & $2.2 \times \mathrm{e}^{-16}$ \\
OLL-RR & 0.1553 & 1.2119 & 0.6550 & $2.2 \times \mathrm{e}^{-16}$ \\
RW & 0.1090 & 1.2120 & 0.0874 & 0.4282 \\
K-RW & 0.0812 & 0.7657 & 0.0759 & 0.6118 \\
E-RW & 0.1091 & 0.7658 & 0.0874 & 0.4287 \\
BRW & 0.0809 & 0.6207 & 0.0757 & 0.6147 \\
T-RW & 0.0871 & 0.6209 & 0.0782 & 0.5734 \\
MO-RW & 0.0886 & 0.6142 & 0.0763 & 0.5168 \\
Mc-RW & 0.1333 & 1.0608 & 0.0807 & 0.5332 \\
\hline
\end{tabular}


Table 3

\begin{tabular}{|c|c|c|c|c|c|}
\hline Model & $\begin{array}{c}\text { Estimates } \\
\widehat{a_{1}}\end{array}$ & $\widehat{a_{2}}$ & $\hat{c}$ & $\widehat{\beta}$ & $\hat{b}$ \\
\hline GOLL-RR & $\begin{array}{l}2.4703 \\
(0.211)\end{array}$ & $\begin{array}{l}1.6377 \\
(0.000)\end{array}$ & & $\begin{array}{l}1.0039 \\
(0.000)\end{array}$ & \\
\hline OLLE-RW & $\begin{array}{l}0.1351 \\
(0.011)\end{array}$ & & $\begin{array}{l}3.7216 \\
(0.0034)\end{array}$ & $\begin{array}{l}0.9296 \\
(0.0033)\end{array}$ & $\begin{array}{l}21.319 \\
(0.0034)\end{array}$ \\
\hline OLLE-RR & $\begin{array}{l}0.4946 \\
(0.0414)\end{array}$ & & $\begin{array}{l}0.067 \\
(0.7195)\end{array}$ & $\begin{array}{l}1.74262 \\
(9.301)\end{array}$ & \\
\hline OLL-RR & $\begin{array}{l}0.49459 \\
0.04135\end{array}$ & & & $\begin{array}{l}0.4524 \\
0.03869\end{array}$ & \\
\hline RW & & & & $\begin{array}{l}1.3968 \\
(0.0336)\end{array}$ & $\begin{array}{l}4.3724 \\
(0.3278)\end{array}$ \\
\hline K-RW & & $\begin{array}{l}0.8489 \\
(16.083)\end{array}$ & $\begin{array}{l}1.6239 \\
(0.6979)\end{array}$ & $\begin{array}{l}1.6341 \\
(9.049)\end{array}$ & $\begin{array}{l}3.4208 \\
(0.7635)\end{array}$ \\
\hline E-RW & & $\begin{array}{l}0.9395 \\
(3.543)\end{array}$ & & $\begin{array}{l}1.4169 \\
(2.568)\end{array}$ & $\begin{array}{l}0.9395 \\
(0.3278)\end{array}$ \\
\hline B-RW & & $\begin{array}{l}0.7346 \\
(1.5290)\end{array}$ & $\begin{array}{l}1.5830 \\
(0.7132)\end{array}$ & $\begin{array}{l}1.6684 \\
(0.7662)\end{array}$ & $\begin{array}{l}3.5112 \\
(0.9683)\end{array}$ \\
\hline T-RW & $\begin{array}{l}-0.7166 \\
(0.2616)\end{array}$ & & & $\begin{array}{l}1.2656 \\
(0.0579)\end{array}$ & $\begin{array}{l}4.7121 \\
(0.3657)\end{array}$ \\
\hline MO-RW & & $\begin{array}{l}0.0033 \\
(0.0009)\end{array}$ & & $\begin{array}{l}6.2296 \\
(1.0134)\end{array}$ & $\begin{array}{l}1.2419 \\
(0.1181)\end{array}$ \\
\hline Mc-RW & $\begin{array}{l}0.8503 \\
(0.1353)\end{array}$ & $\begin{array}{l}44.423 \\
(25.100)\end{array}$ & $\begin{array}{l}19.859 \\
(6.706)\end{array}$ & $\begin{array}{l}0.0203 \\
(0.0060)\end{array}$ & $\begin{array}{l}46.974 \\
(21.871)\end{array}$ \\
\hline
\end{tabular}

Table 4

\begin{tabular}{|c|c|c|c|c|}
\hline \multirow[t]{2}{*}{ Model } & \multicolumn{4}{|c|}{ Goodness of fit criteria } \\
\hline & C-V-M & A-D & K-S & $\mathrm{P}$-value \\
\hline GOLL-RR & 0.0525 & 0.4530 & 0.0703 & 0.8926 \\
\hline OLLE-RW & 0.1049 & 0.8325 & 0.5520 & $6.7 \times \mathrm{e}^{-16}$ \\
\hline OLLE-RR & 0.1502 & 1.1469 & 0.6795 & $6.7 \times \mathrm{e}^{-16}$ \\
\hline OLL-RR & 0.1502 & 1.1469 & 0.6795 & $6.7 \times \mathrm{e}^{-16}$ \\
\hline RW & 0.0707 & 0.5332 & 0.0772 & 0.8185 \\
\hline K-RW & 0.0634 & 0.4981 & 0.0715 & 0.8810 \\
\hline E-RW & 0.0707 & 0.5332 & 0.0772 & 0.8187 \\
\hline B-RW & 0.0640 & 0.5008 & 0.0716 & 0.8804 \\
\hline T-RW & 0.0655 & 0.4939 & 0.0735 & 0.8470 \\
\hline MO-RW & 0.0629 & 0.4902 & 0.0813 & 0.7685 \\
\hline Mc-RW & 0.1161 & 0.9193 & 0.0831 & 0.7455 \\
\hline
\end{tabular}


Table 5

\begin{tabular}{llllll}
\hline Model & $\begin{array}{c}\text { Estimates } \\
\widehat{a_{1}}\end{array}$ & \multicolumn{1}{c}{$\widehat{a_{2}}$} & \multicolumn{1}{c}{$\hat{c}$} & \multicolumn{1}{c}{$\hat{\beta}$} & \multicolumn{1}{c}{$\hat{b}$} \\
& 3.032 & 2.1568 & & 0.86599 & \\
GOLL-RR & $(0.325)$ & $(9.145)$ & & $(1.836)$ & \\
& 0.1449 & & 0.00879 & 1.2997 & 24.878 \\
OLLE-RW & $(0.0129)$ & & $(0.000)$ & $(0.000)$ & $(0.000)$ \\
& 0.5025 & & 0.0716 & 1.7048 & \\
OLLE-RR & $(0.0529)$ & & $(1.13062)$ & $(13.47)$ & \\
& 0.50251 & & & 0.45599 & \\
OLL-RR & 0.05295 & & & 0.048652 & \\
& & & & 1.4108 & 5.4377 \\
RW & & & & $(0.0344)$ & $(0.5192)$ \\
& & 0.2855 & 1.2824 & 1.9142 & 4.7731 \\
K-RW & & $(9.1338)$ & $(0.6388)$ & $(12.836)$ & $(1.3134)$ \\
& & 0.9059 & & 1.4367 & 5.4379 \\
E-RW & & $(2.764)$ & & $(4.324)$ & $(0.5193)$ \\
& & 1.2996 & 1.2649 & 1.3945 & 4.7927 \\
BRW & & $(4.4378)$ & $(0.6640)$ & $(0.9304)$ & $(1.4641)$ \\
& & & & 1.5491 & 4.3139 \\
T-RW & 0.7778 & & & $(0.0655)$ & $(0.5849)$ \\
& $(0.2477)$ & & & 5.2383 & 1.4537 \\
MO-RW & & 0.0023 & & $(0.8209)$ & $(0.1650)$ \\
& & $(0.0004)$ & & 0.0073 & 29.104 \\
Mc-RW & & 56.227 & 14.953 & $(0.0013)$ & $(11.304)$ \\
\hline & & $(30.539)$ & $(4.733)$ & & \\
\hline
\end{tabular}

Table 6

\begin{tabular}{lllll}
\hline Model & \multicolumn{4}{l}{ Goodness of fit criteria } \\
& C-V-M & A-D & K-S & P-value \\
\hline GOLL-RR & 0.19551 & 1.3498 & 0.11008 & 0.5797 \\
\hline RW & 0.3233 & 2.0301 & 0.1506 & 0.2066 \\
E-RW & 0.3233 & 2.0301 & 0.1506 & 0.2064 \\
T-RW & 0.2823 & 1.8152 & 0.1370 & 0.3045 \\
\hline
\end{tabular}

Table 7

\begin{tabular}{|c|c|c|c|c|c|}
\hline Model & $\begin{array}{c}\text { Estimates } \\
\widehat{a_{1}}\end{array}$ & $\widehat{a_{2}}$ & $\hat{c}$ & $\widehat{\beta}$ & $\hat{b}$ \\
\hline GOLL-RR & $\begin{array}{l}1.961 \\
(0.234)\end{array}$ & $\begin{array}{l}0.111 \\
(0.000)\end{array}$ & & $\begin{array}{l}1.4123 \\
(0.000)\end{array}$ & \\
\hline RW & & & & $\begin{array}{l}0.4859 \\
(0.023)\end{array}$ & $\begin{array}{l}3.2078 \\
(0.3263)\end{array}$ \\
\hline E-RW & & & $\begin{array}{l}0.9047 \\
(18.784)\end{array}$ & $\begin{array}{l}0.5013 \\
(3.244)\end{array}$ & $\begin{array}{l}3.2077 \\
(0.3263)\end{array}$ \\
\hline T-RW & $\begin{array}{l}-0.5816 \\
(0.2787)\end{array}$ & & & $\begin{array}{l}0.4400 \\
(0.0290)\end{array}$ & $\begin{array}{l}3.4974 \\
(0.3527)\end{array}$ \\
\hline
\end{tabular}



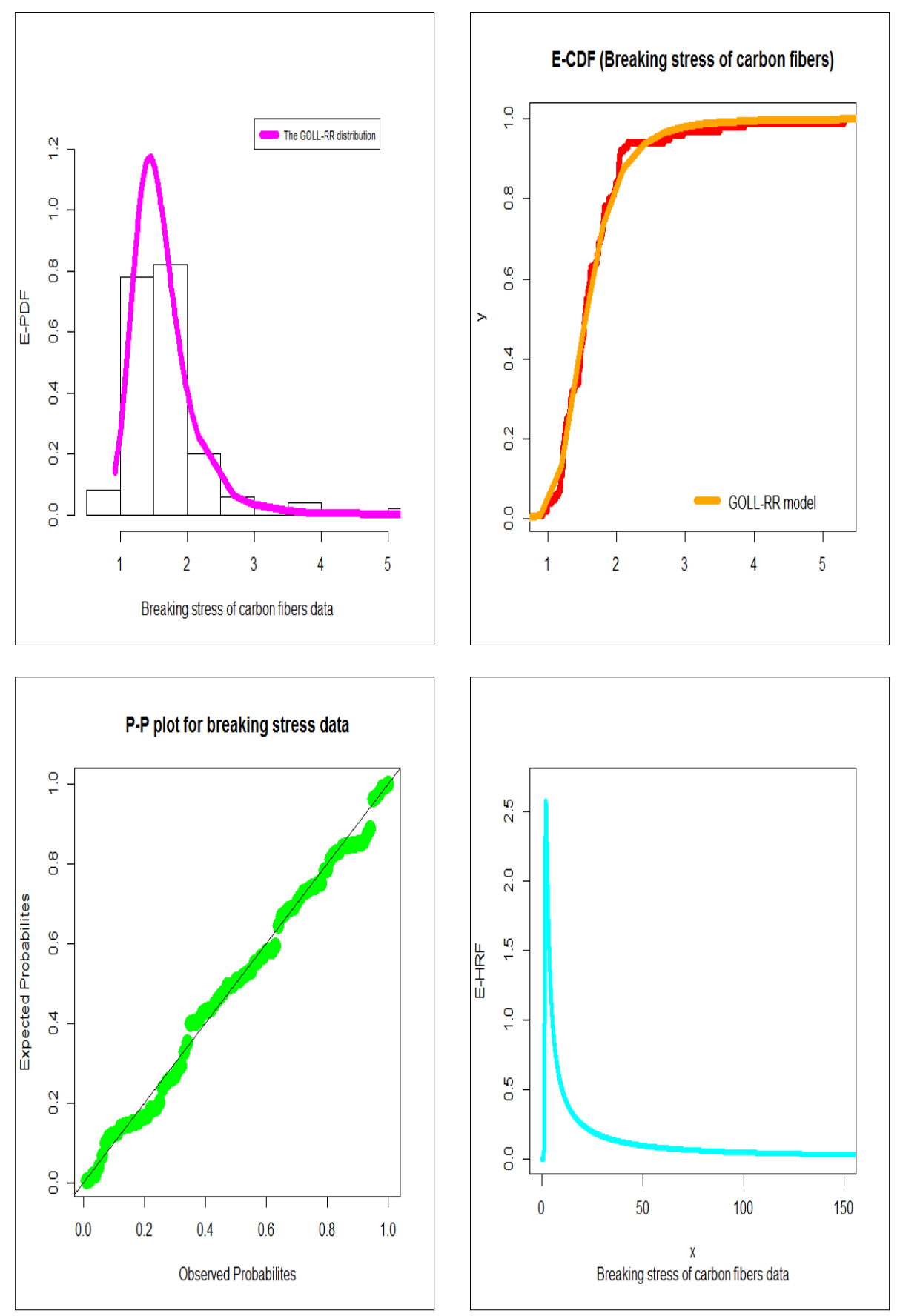

Fig. 5: E-PDF, E-CDF, P-P plot and E-HRF for data set I. 

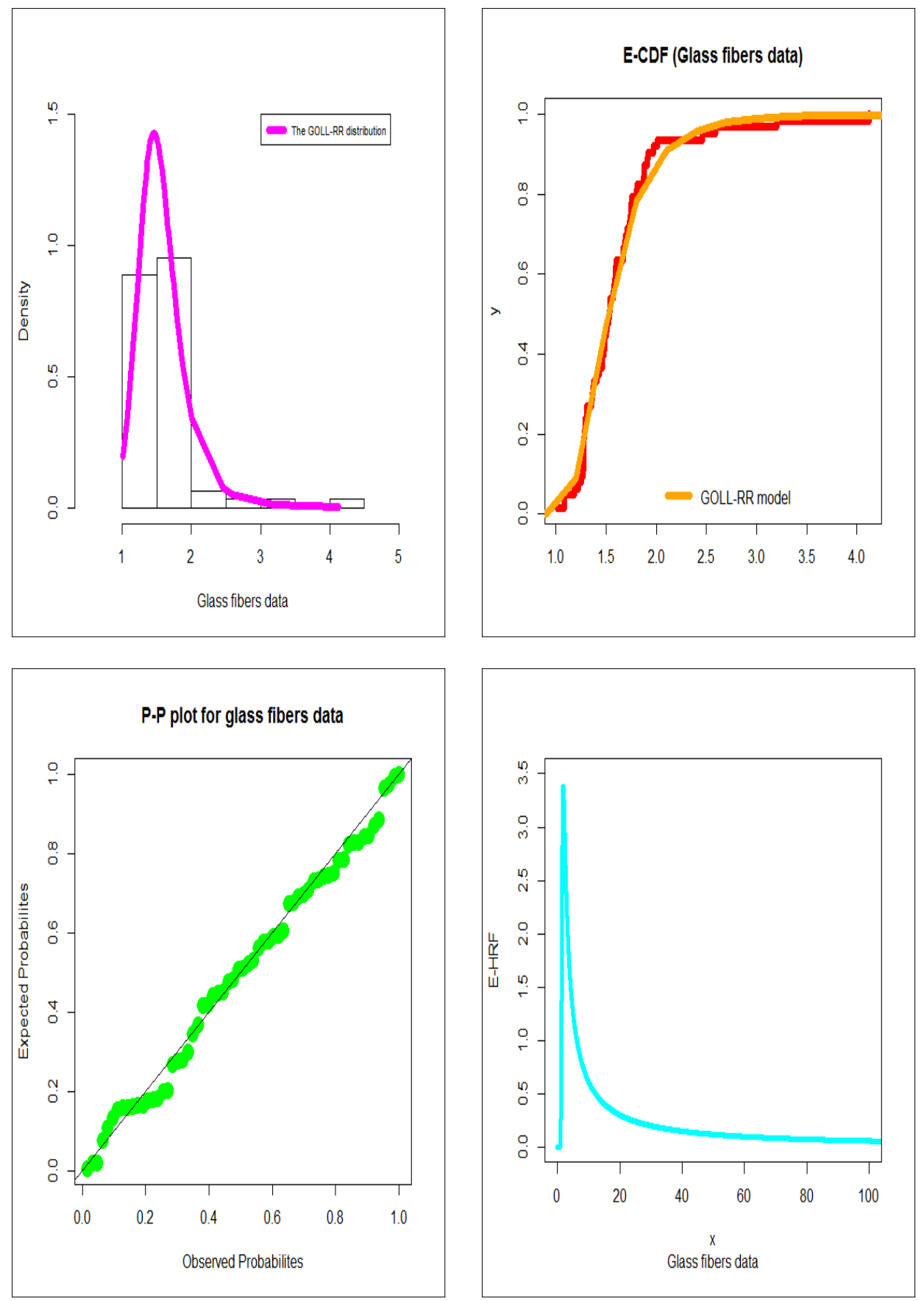

Fig. 6: E-PDF, E-CDF, P-P plot and E-HRF for data set II. 

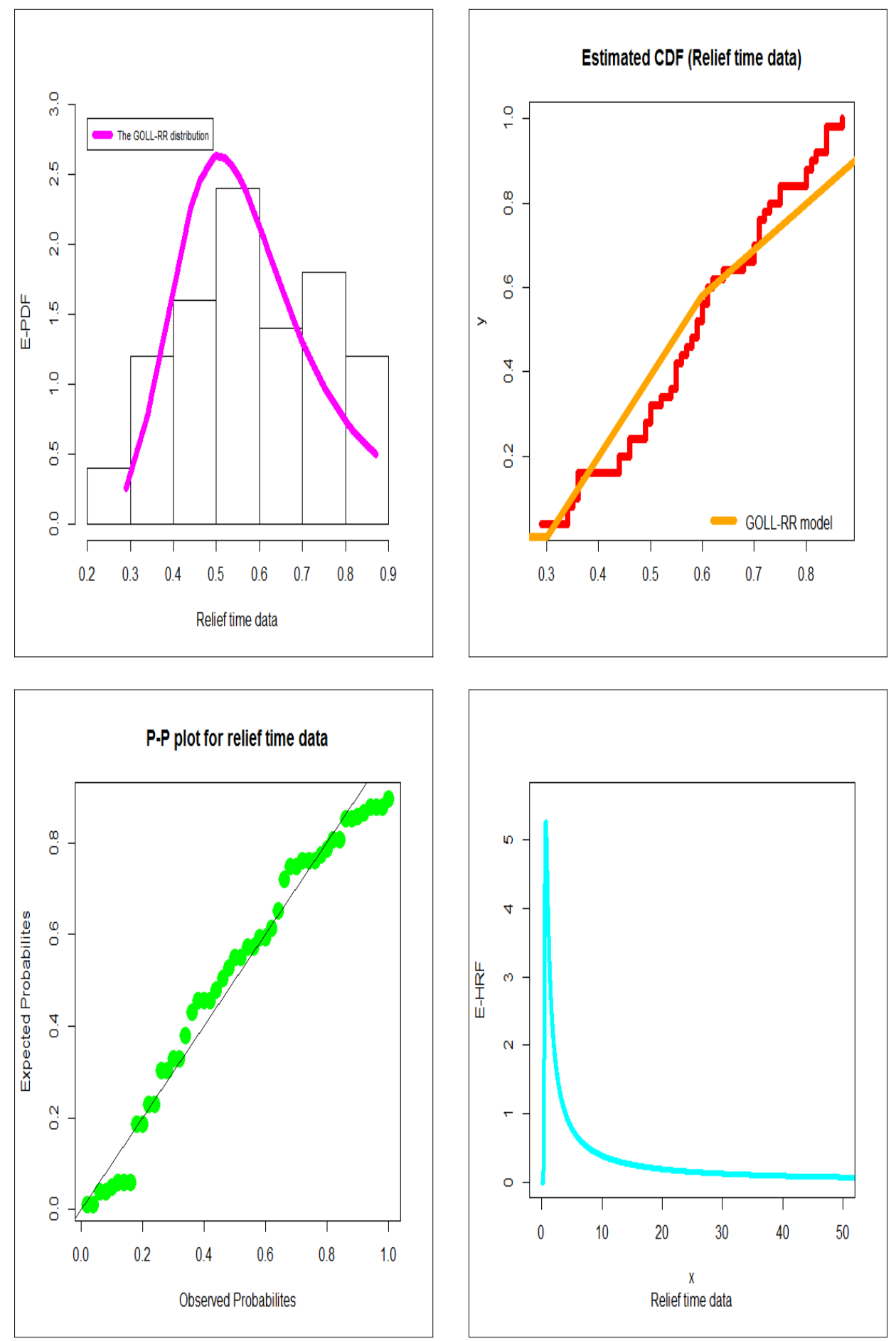

Fig. 7: E-PDF, E-CDF, P-P plot and E-HRF for data set III 


\section{Concluding remarks}

A new extension of the reciprocal Rayleigh distribution is introduced. Simple type copula-based construction is presented for deriving and many multivariate and bivariate type reciprocal Rayleigh models. The new PDF can is expressed as a "double linear mixture" of the reciprocal Rayleigh PDF. The new reciprocal Rayleigh model generalizes another three reciprocal Rayleigh distributions. The performance of the estimation method is assessed using a graphical simulation.

\section{Acknowledgement}

This research was funded by the Deanship of Scientific Research at Princess Nourah bint Abdulrahman University through the Fast-track Research Funding Program.

\section{References}

1. Aarset, M. V. (1987). How to identify a bathtub hazard rate. IEEE Transactions on Reliability, 36(1), 106-108.

2. Alizadeh, M., Ghosh, I., Yousof, H. M., Rasekhi, M. and Hamedani G. G. (2017). The generalized odd generalized exponential family of distributions: properties, characterizations and applications, J. Data Sci. 15, 443-466.

3. Alizadeh, M., Rasekhi, M., Yousof, H. M. and Hamedani G. G. (2018). The transmuted Weibull G family of distributions. Hacettepe Journal of Mathematics and Statistics, 47(6), 1-20.

4. Alizadeh, M., Yousof, H. M. Rasekhi, M. and Altun, E. (2019). The odd log-logistic Poisson-G Family of distributions, Journal of Mathematical Extensions, 12(3), 81-104.

5. Aryal, G. R. and Yousof, H. M. (2017). The exponentiated generalized-G Poisson family of distributions. Economic Quality Control, 32(1), 1-17.

6. Barreto-Souza, W. M., Cordeiro, G. M. and Simas, A. B. (2011). Some results for beta Fréchet distribution. Commun. Statist. Theory-Meth., 40, 798-811.

7. Brito, E., Cordeiro, G. M., Yousof, H. M., Alizadeh, M. and Silva, G. O. (2017). Topp-Leone Odd Log-Logistic Family of Distributions, Journal of Statistical Computation and Simulation, 87(15), 3040-3058.

8. Cordeiro, G. M., Alizadeh, M.M., Ozel, G., Hosseini, B., Ortega, E. M. M. and Altun, E. (2016). The generalized odd log-logistic family of distributions: properties, regression models and applications, Journal of Statistical Computation and Simulation, 87(5), 908-932.

9. Cordeiro, G. M., Yousof, H. M., Ramires, T. G. and Ortega, E. M. M. (2018). The Burr XII system of densities: properties, regression model and applications. Journal of Statistical Computation and Simulation, 88(3), 432456.

10. Fréchet, M. (1927). Sur la loi de probabilité de lécart maximum. Ann. de la Soc. polonaisede Math, 6, 93-116.

11. Goual, H. and Yousof, H. M. (2019). Validation of Burr XII inverse Rayleigh model via a modified chi-squared goodness-of-fit test. Journal of Applied Statistics, 47(1), 1-32.

12. Goual, H., Yousof, H. M. and Ali, M. M. (2020). Lomax inverse Weibull model: properties, applications and a modified chi-squared goodness-of-fit test for validation. J. Nonlinear Sci. Appl., forthcoming.

13. Gusmao, F. R. S., Ortega, E. M. M. and Cordeiro, G. M. (2011). The generalized inverse Weibull distribution. Statistical Papers 52, 591-619.

14. Gleaton, J. U. and Lynch, J.D. (2006). Properties of generalized loglogistic families of lifetime distributions. Journal of Probability and Statistical Science, 4, 51-64.

15. Gupta, R. C. and Gupta, R. D. (2007). Proportional reversed hazard rate model and its applications. J Statist Plan Inference.137, 3525-3536.

16. Hamedani G. G., Altun, E, Korkmaz, M. C., Yousof, H. M. and Butt, N. S. (2018). A new extended G family of continuous distributions with mathematical properties, characterizations and regression modeling. Pak. J. Stat. Oper. Res., 14(3), 737-758.

17. Hamedani G. G. Rasekhi, M., Najibi, S. M., Yousof, H. M. and Alizadeh, M., (2019). Type II general exponential class of distributions. Pak. J. Stat. Oper. Res., 15 (2), 503-523.

18. Hamedani G. G. Yousof, H. M., Rasekhi, M., Alizadeh, M., Najibi, S. M. (2017). Type I general exponential class of distributions. Pak. J. Stat. Oper. Res., 14(1), 39-55.

19. Ibrahim, M. (2019). A new extended Fréchet distribution: properties and estimation. Pak. J. Stat. Oper. Res.,15 (3), 773-796. 
20. Ibrahim, M. (2020a). The compound Poisson Rayleigh Burr XII distribution: properties and applications. Journal of Applied Probability and Statistics, forthcoming.

21. Ibrahim, M. (2020b). The generalized odd log-logistic Nadarajah Haghighi distribution: statistical properties and different methods of estimation. Journal of Applied Probability and Statistics, forthcoming.

22. Ibrahim, M., Goual, H., Alotaibi, R., Ali, M.M. Rezk, H. R. and Yousof, H. M. (2020). Censored and uncensored validation for the double Burr XII model using a new Nikulin-Rao-Robson goodness-of-fit test with Bayesian and non-Bayesian estimation, Journal of Taibah University for Science, forthcoming.

23. Ibrahim, M., Yadav, A. S. Yousof, H. M., Goual, H. and Hamedani, G. G. (2019). A new extension of Lindley distribution: modified validation test, characterizations and different methods of estimation, Communications for Statistical Applications and Methods, 26(5), 473-495.

24. Korkmaz, M. C., Altun, E., Yousof, H. M. and Hamedani G. G. (2019). The odd power Lindley generator of probability distributions: properties, characterizations and regression modeling, International Journal of Statistics and Probability, 8(2), 70-89.

25. Korkmaz, M. C. Yousof, H. M. and Ali, M. M. (2017). Some theoretical and computational aspects of the odd Lindley Fréchet distribution, Journal of Statisticians: Statistics and Actuarial Sciences, 2, 129-140.

26. Korkmaz, M. C. Yousof, H. M. and Hamedani G. G. (2018). The exponential Lindley odd log-logistic G family: properties, characterizations and applications. Journal of Statistical Theory and Applications, 17(3), 554 - 571.

27. Mahmoud, M. R. and Mandouh, R. M. (2013). On the transmuted Fréchet distribution. Journal of Applied Sciences Research, 9, 5553-5561.

28. Merovci, F., Alizadeh, M., Yousof, H. M. and Hamedani G. G. (2017). The exponentiated transmuted-G family of distributions: theory and applications, Communications in Statistics-Theory and Methods, 46(21), 1080010822.

29. Nascimento, A. D. C., Silva, K. F., Cordeiro, G. M., Alizadeh, M. and Yousof, H. M. (2019). The odd NadarajahHaghighi family of distributions: properties and applications. Studia Scientiarum Mathematicarum Hungarica, $56(2), 1-26$.

30. Nichols, M. D, Padgett, W. J. (2006). A Bootstrap control chart for Weibull percentiles. Quality and Reliability Engineering International, 22, 141-151.

31. Smith, R. L. and Naylor, J. C. (1987). A comparison of maximum likelihood and bayesian estimators for the three-parameter Weibull distribution. Applied Statistics, 36, 358-369.

32. Yadav, A. S., Goual, H., Alotaibi, R. M. Rezk, H., Ali, M. M. and Yousof, H. M. (2020). Validation of the ToppLeone-Lomax model via a modified Nikulin-Rao-Robson goodness-of-fit test with different methods of estimation. Symmetry, 12, 1-26. doi: 10.3390/sym12010057

33. Yousof, H. M., Afify, A. Z., Alizadeh, M., Nadarajah, S., Aryal, G. R. and Hamedani, G. G. (2018a). The Marshall-Olkin generalized-G family of distributions with Applications, STATISTICA, 78(3), 273- 295.

34. Yousof, H. M., Altun, E. and Hamedani, G. G. (2018b). A new extension of Frechet distribution with regression models, residual analysis and characterizations. Journal of Data Science, 16(4), 743-770.

35. Yousof, H. M., Afify, A. Z., Alizadeh, M., Hamedani G. G., Jahanshahi, S. M. A. and Ghosh, I. (2018c). The generalized transmuted Poisson-G family of Distributions. Pak. J. Stat. Oper. Res., 14 (4), 759-779.

36. Yousof, H. M., Altun, E., Ramires, T. G., Alizadeh, M. and Rasekhi, M. (2018c). A new family of distributions with properties, regression models and applications, Journal of Statistics and Management Systems, 21(1), 163188.

37. Yousof, H. M., Butt, N. S., Alotaibi, R., Rezk, H. R., Alomani, G. A. and Ibrahim, M. (2019). A new compound Fréchet distribution for modeling breaking stress and strengths data. Pak. J. Stat. Oper. Res., 15 (4), 1017-1035.

38. Yousof, H. M., Rasekhi, M., Altun, E. and Alizadeh, M. (2018d). The extended odd Frechet family of distributions: properties, applications and regression modeling. International Journal of Applied Mathematics and Statistics, 30(1), 1-30. 